\title{
Identification of Differentially Expressed Genes in Brassinosteroid-Treated Brassica napus Seedlings
}

\author{
Sangeeta Dhaubhadel · Priti Krishna
}

Received: 17 January 2008/ Accepted: 20 May 2008/Published online: 12 July 2008

(C) The Author(s) 2008

\begin{abstract}
Brassinosteroid-mediated gene expression changes have been reported primarily in Arabidopsis thaliana but not in its close relative Brassica napus. To obtain an initial idea of the molecular changes induced by longterm exposure to 24-epibrassinolide (EBR) in B. napus seedlings, we used the differential display-reverse transcription PCR technique. Six differentially expressed cDNAs were isolated and characterized. These encode a mitochondrial transcription termination factor (mTERF)related protein, glycine-rich protein 22 (GRP22), myrosinase, 3-ketoacyl-CoA thiolase, and a copia-like polyprotein. The first four were upregulated in EBR-treated seedlings while the latter was expressed at higher levels in untreated seedlings. Transcripts of mTERF-related protein, GRP22, and myrosinase were present at higher levels in treated seedlings under nonstress conditions, whereas those of 3ketoacyl-CoA thiolase rose to higher levels in treated seedlings during exposure to heat stress. The results of the present study indicate that EBR treatment in B. napus leads to substantial changes in the expression levels of genes involved in a variety of physiologic responses. The results provide a useful framework for further research into EBRmediated molecular changes in B. napus, which will also
\end{abstract}

S. Dhaubhadel · P. Krishna $(\square)$

Department of Biology, University of Western Ontario, 1151

Richmond St. North, London, ON, Canada N6A 5B7

e-mail: pkrishna@uwo.ca

Present Address:

S. Dhaubhadel

Agriculture and Agri-Food Canada, 1391 Sandford Street,

London, ON, Canada N5V 4T3

e-mail: dhaubhadels@agr.gc.ca add to our understanding of how brassinosteroids mediate stress tolerance in this agriculturally important oil crop.

Keywords Brassica napus - Brassinosteroid . Gene expression $\cdot$ Stress tolerance

\section{Introduction}

Brassinosteroids (BRs) are a group of naturally occurring polyhydroxy steroids in plants that regulate a wide range of growth responses, including cell elongation and proliferation, vascular differentiation, and reproduction (Sasse 2003), and also modulate plant responses to various environmental stresses (Krishna 2003). The study of Arabidopsis, pea, tomato, and rice mutants defective in BR biosynthesis has revealed the crucial role of BRs in plant growth and development, and has provided a good understanding of the BR biosynthetic pathways (Clouse and Sasse 1998; Bishop 2003). In recent years, several BR signaling components have also been identified. These include the BRI1 and BAK1 receptor-like kinases, BKI1 (BRI1 kinase inhibitor 1), the GSK3/SHAGGY-like kinase BIN2 that negatively regulates BR signaling, a nuclear localized phosphatase BSU1, and transcription factors BES1 and BZR1 that mediate major BR effects (Belkhadir and others 2006; Karlova and others 2006). According to the current model of BR signaling, BR binding to BRI1 leads to rapid dissociation of BRI1 from BKI1 followed by hetero-oligomerization and activation of BRI1 and BAK1 (Li and Jin 2007). In the next step(s) the phosphorylation activity of BIN2 is inhibited, possibly through a membrane recruitment mechanism. As a result, BES1 and BZR1 are rapidly dephosphorylated, most likely by BSU1, allowing them to homodimerize or combine with other transcription factors to bind to promoters of 
BR-responsive genes. BAK1 and a related protein, in addition to positively regulating the BR-dependent growth pathway, also negatively regulate a BR-independent celldeath pathway (He and others 2007).

The identification of genes regulated by BRs is requisite for understanding how BRs regulate a wide variety of physiologic processes. Genes responsive to BRs at the transcriptional level encode enzymes and proteins involved in cell wall loosening (Goda and others 2002; Mussig and others 2002), regulation of cell division (Hu and others 2000), carbohydrate metabolism (Goetz and others 2000), ethylene (Yi and others 1999) and jasmonic acid biosynthesis (Mussig and others 2000) protein translation (Jiang and Clouse 2001; Dhaubhadel and others 2002), and other physiologic processes (Mussig and others 2006). In addition to analyzing gene expression on a small scale, several recent studies have employed DNA microarrays to identify BR-regulated genes (Goda and others 2002; Mussig and others 2002; Yin and others 2002; Vert and others 2005). Some of these genes are regulated specifically by BRs, whereas others show regulation by additional phytohormones such as auxin. Most of these studies have focused on the effects of short-term treatment with BR. A common observation is that BR effects on gene expression in Arabidopsis are small in magnitude.

We have been studying the effects of long-term treatment with 24-epibrassinolide (EBR), a BR, on the growth and heat stress response of Brassica napus. Our initial studies involving uptake of EBR by roots indicated that 57 days' exposure to EBR was required to see the stresstolerant phenotype. We have previously demonstrated that $B$. napus seedlings grown in the presence of EBR for 14 days accumulate higher mRNA and protein levels of the major classes of heat shock proteins (hsps) during and after heat stress compared with untreated seedlings (Dhaubhadel and others 1999), and that a modified translation machinery is responsible for higher hsp synthesis during heat stress and more rapid resumption of cellular protein synthesis following stress (Dhaubhadel and others 2002). In addition to these, what other molecular changes are induced by EBR in B. napus seedlings is currently not known.

The present study was conducted to shed light on gene expression changes in EBR-treated B. napus, a close relative of Arabidopsis and an important oil seed crop. Keeping in mind that BR-mediated changes at the transcript level are relatively small; we avoided using Arabidopsis microarrays and instead used the differential display method to examine mRNA expression profiles in EBR-treated and untreated B. napus seedlings. Of the differentially expressed cDNAs that were isolated, six cDNAs were studied for their sequences and expression patterns. The results of these experiments suggest that EBR treatment of B. napus leads to substantial increases in the expression of genes involved in a variety of physiological responses.

\section{Materials and Methods}

Plant Material and Growth Conditions

Plant material, growth conditions, and heat stress were as described previously (Dhaubhadel and others 1999). EBR was obtained from CIDtech (Cambridge, Ontario, Canada).

\section{DD-RT PCR}

Total RNA was isolated from 14-day-old untreated and EBR-treated B. napus seedlings that were heat stressed at $45^{\circ} \mathrm{C}$ for 1,2 or $3 \mathrm{~h}$, using the method described by Glisin and others (1974) with some modifications. The frozen tissue was ground in liquid nitrogen, the powder was transferred to 5 volumes of guanidine isothiocyanate (GITC) homogenization buffer (4.0 M GITC, 0.1 M Tris$\mathrm{HCl} \mathrm{pH} \mathrm{7.5,} \mathrm{and} 1 \% \beta$-mercaptoethanol) and homogenized, and sodium lauryl sarcosinate was added to a final concentration of $0.5 \%$. The sample was clarified by centrifugation, layered onto a $1.2-\mathrm{ml}$ cushion of $5.7 \mathrm{M}$ $\mathrm{CsCl}$ and $0.01 \mathrm{M}$ EDTA pH 7.5, and centrifuged at $20^{\circ} \mathrm{C}$ for $18 \mathrm{~h}$ at 35,000 rpm in a Ti 50.1 Beckmann rotor. The RNA pellet was washed with ethanol, dissolved in TE buffer, and then precipitated.

The RNA was treated with DNase 1 and reverse-transcribed using RNAimage kits (GenHunter, Nashville, TN). RNA $(0.2 \mu \mathrm{g})$ was reverse-transcribed in the presence of $20 \mu \mathrm{M}$ dNTPs and $0.2 \mu \mathrm{M}$ anchor primer (5'-AAGC TTTTTTTTTTTA- $3^{\prime}, 5^{\prime}$-AAGCTTTTTTTTTTTC- ${ }^{\prime}$, or $5^{\prime}$-AAGCTTTTTTTTTTTG- $3^{\prime}$ ) for $1 \mathrm{~h}$. Two microliters of the reaction was PCR-amplified with the same anchor primer and $0.2 \mu \mathrm{M}$ arbitrary primer provided in the kit $(\mathrm{H}$ AP1 to H-AP16) in the presence of $2 \mu \mathrm{M}$ dNTPs and $1 \mu \mathrm{l}$ $\left[\alpha_{-}{ }^{35} \mathrm{~S}\right] \mathrm{dATP}(1000 \mathrm{Ci} / \mathrm{mmol}$, Amersham). Amplification was carried out as follows: $94^{\circ} \mathrm{C}$ for $15 \mathrm{~s}, 40^{\circ} \mathrm{C}$ for $2 \mathrm{~min}$, and $72^{\circ} \mathrm{C}$ for $30 \mathrm{~s}$ (40 cycles) followed by $72^{\circ} \mathrm{C}$ for 5 min. PCR products were separated on a $6 \%$ denaturing polyacrylamide gel and visualized by autoradiography. Differentially expressed cDNAs were excised and reamplified using the same PCR conditions, with the exception that the dNTP concentrations were increased to $20 \mu \mathrm{M}$ and no radioisotope was added.

\section{Extension of $5^{\prime}$ Sequence Using $5^{\prime}$ RACE}

The nucleotide sequences of the cloned cDNAs were determined on both strands. Based on sequence information, three gene-specific primers (GSP1, GSP2, and GSP3) were designed for each of the five cDNAs using the Oligo calculator program (DNAStar).

For $5^{\prime}$ extension of the sequences of interest, mRNA $(0.5 \mu \mathrm{g})$ isolated from EBR-treated seedlings was reverse- 
transcribed using $1 \mu \mathrm{g}$ of random hexamers, $0.5 \mathrm{mM}$ of each dNTP, and 200 units of Superscript II reverse transcriptase (Gibco, BRL). The first-strand product was purified using the QIAquick PCR Purification Kit (Qiagen, Valencia, CA), and a dA-tail was attached to it using terminal deoxynucleotidyl transferase. The second-strand synthesis was carried out using purified dA-tailed cDNA, $1 \mu \mathrm{g}$ of anchor dT primer (5'-TGACTGGACTATGGTCTCGACATGGCGTAGTCG TTTTTTTTTTTTTTTTTT-3' ${ }^{\prime}$ ), $0.5 \mathrm{mM}$ of dNTP, and 200 units of Superscript II reverse transcriptase for $1 \mathrm{~h}$. The double-stranded product was purified again and then used for PCR amplification with GSP1 and each of the 5'-RACEspecific anchor primers (anchor primer1: 5'-TGACTGG ACTATGGTCTC-3', anchor primer2: 5'-CTCGACATGG CGTAGTC-3', anchor primer3: 5'-GGTCTCGACATGGC GTA-3', anchor primer4: 5'-GGTCTCGACATGGCGTAG TCG-3', anchor primer5: 5'-GGTCTCGACATGGCGTAG TCGT $-3^{\prime}$ ). The primary PCR reaction was diluted 50 times and used for secondary nested PCR, using GSP2 primer and different anchor primers. The secondary nested PCR reaction was again diluted 50 times and used for tertiary nested PCR, using GSP3 primer and anchor primers. After each PCR, the PCR products were analyzed on an agarose gel against the appropriate molecular size markers. The final confirmation was carried out by Southern blotting, using radiolabeled differentials as probes. The $5^{\prime}$-RACE products were cloned into the TA cloning vector and sequenced at the Robarts Sequencing Facility, London, Ontario.

The extension of SD19C was not successful using $5^{\prime}$ RACE. A conserved sequence ( $5^{\prime}$-AAGGAAAGGTGAG TAGGGGAGTGA-3') toward the $5^{\prime}$ end of myrosinase genes was identified by alignment of seven different myrosinase gene sequences. Following reverse transcription, a SD19C gene-specific primer (5'-GATAGAACAAACTCC TTATTATGAAGC- $3^{\prime}$ ) and the conserved sequence primer were used for amplification by PCR $\left[94^{\circ} \mathrm{C}\right.$ for $1 \mathrm{~min}, 60^{\circ} \mathrm{C}$ for $40 \mathrm{~s}$, and $72^{\circ} \mathrm{C}$ for $3 \mathrm{~min}$ (35 cycles)]. The RT-PCR product was cloned into the TA cloning vector.

\section{Sequence Analysis}

DNA and deduced amino acid sequences were analyzed using the BLAST program (Altschul and others 1997) set to standard parameters. DNAMAN was used to determine molecular mass, isoelectric point, and hydrophobicity of the polypeptide encoded by the SD7C clone.

\section{Northern Blot Analysis}

Northern blot hybridization was carried out as described previously (Dhaubhadel and others 1999). Following hybridization, the blots were washed twice at room temperature with $1 \times \mathrm{SSC}, 0.1 \% \mathrm{SDS}$ for $15 \mathrm{~min}$, then once with $0.25 \times \mathrm{SSC}, 0.1 \% \mathrm{SDS}$ at $55^{\circ} \mathrm{C}$ for $15 \mathrm{~min}$. cDNAs that were confirmed as differentials were cloned into the pCR2.1 (TA cloning) vector (Invitrogen, Carlsbad, CA). Band intensities were analyzed using a web-based Scion Image program (Scion Corporation, Frederick, MD).

\section{In Silico Gene Expression Analysis}

The Arabidopsis orthologs most closely related to the $B$. napus differentials were identified, and data on their tissuespecific, and abiotic stress and hormone-responsive, gene expression were generated using the AtGenExpress Visualization Tool (AVT) (Schmid and others 2005; Kilian and others 2007; http://jsp.weigelworld.org/expviz/expviz.jsp).

\section{Results}

Screening of Differentially Expressed mRNAs in EBR-Treated B. napus Seedlings

Gene expression profiling in Arabidopsis has indicated that the majority of BR-regulated genes show less than a twofold change in expression in response to BR (Vert and others 2005). To identify gene expression changes induced by long-term exposure to EBR in B. napus, we used the differential display-reverse transcription (DD-RT) PCR technique (Liang and Pardee 1992) versus cross-hybridization of B. napus RNA with Arabidopsis microarrays, and we generated mRNA fingerprints of heat-stressed EBR-treated and untreated B. napus seedlings as opposed to those of unstressed seedlings. These choices were made to ensure signal strengths that would lead to clear identification of genes differentially expressed in response to EBR, as well as in response to EBR and stress. To minimize false positives (Liang and others 1993) and to maximize the probability of isolating genes that are differentially expressed in EBR-treated plants, each reaction was carried out in duplicate, and three time points of heat stress were included in the screen. An example of an mRNA that was differentially expressed at all three time points of heat stress is shown in Figure 1A.

The 80 arbitrary primers provided in the GenHunter RNAimage Kit, when used in combination with three different oligo-dT primers, should detect in the DD screen most mRNAs expressed at any given time in a cell. In the present study, 16 arbitrary primers were used in combination with three anchored primers, which gave rise to 25 differentials. With one exception, all differentials showed higher expression in EBR-treated seedlings than in untreated seedlings. The differentially expressed cDNAs were purified from the gel, reamplified, and used as 
Fig. 1 Identification of differentials in untreated and EBR-treated B. napus seedlings. (A) B. napus seedlings grown for 14 days in the absence $(C)$ or presence (E) of $1 \mu \mathrm{M}$ EBR were exposed to $45^{\circ} \mathrm{C}$ for 1,2 , or $3 \mathrm{~h}$. Total RNA was isolated from both sets of seedlings and subjected to DD-RT PCR. Each reaction was carried out in duplicate. The arrows indicate a cDNA that is differentially expressed in EBR-treated seedlings compared with untreated seedlings at all three time points tested. (B) Northern blot verification of the differential expression of isolated cDNAs. Total RNA used in the DD screen was subjected to Northern blotting. The membrane was hybridized with cloned cDNA fragments. A photograph of the ethidium bromide stained gel is shown below the blot
A

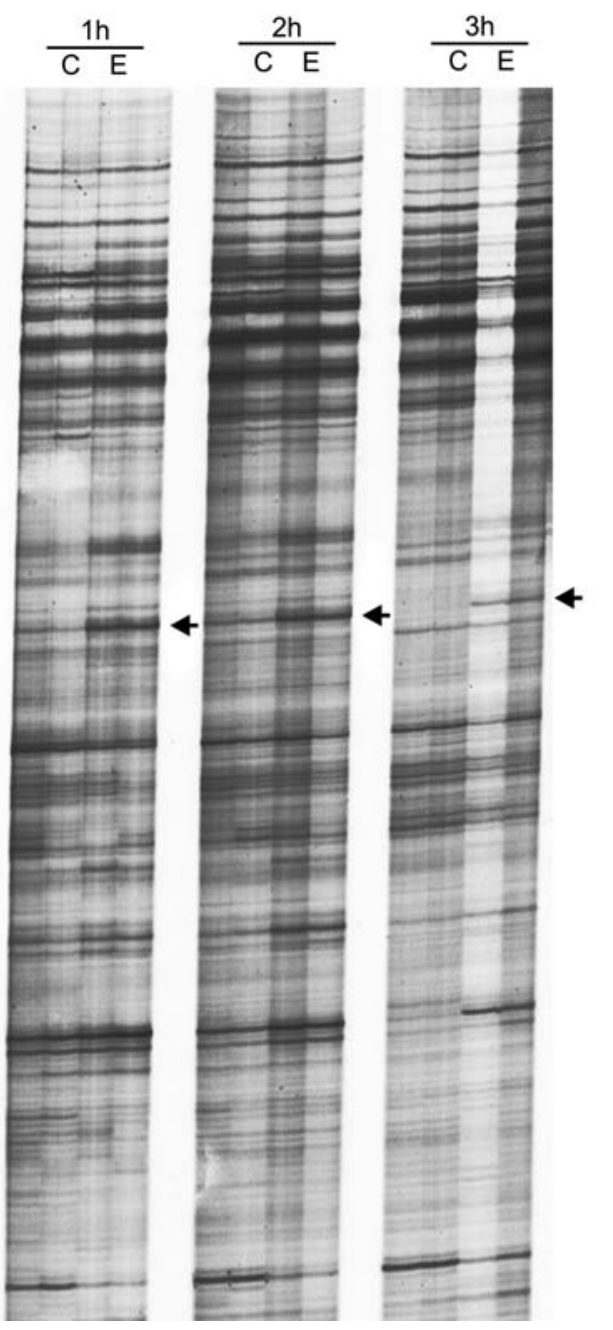

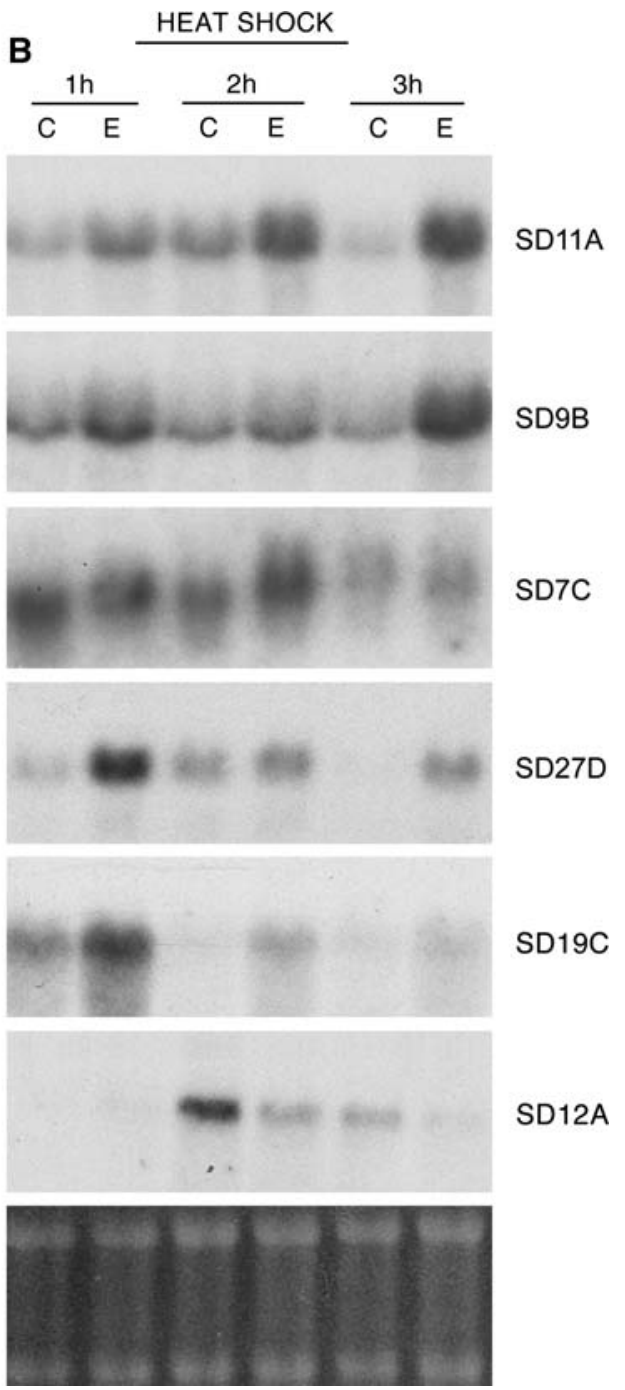

hybridization probes to verify differential expression of corresponding mRNAs. The true differentials were cloned into the TA cloning vector and the differential expression of cloned cDNAs was again confirmed by Northern blot hybridization. Of the 25 differentials that were recovered from the gel, 11 were judged to be true differentials. Finally, six cloned cDNAs that showed significant differences in expression between the two sets of seedlings (Figure 1B) were chosen for further study.

\section{Sequence Analysis of the Differentially Expressed cDNAs}

The nucleotide sequences of the six cDNAs were determined for initial identification of the cDNAs and for generation of gene-specific primers. All six clones were found to contain sequences corresponding to the particular anchor and arbitrary primers used for DD-RT PCR. Genespecific primers were next used to obtain the $5^{\prime}$ sequences of the cDNAs by the $5^{\prime}$-RACE procedure. Sequence analysis of the 5'-RACE products revealed that a full-length sequence of SD7C and partial $5^{\prime}$ sequences of SD11A, SD12A, SD19C, and SD27D cDNAs were obtained by the $5^{\prime}$-RACE procedure. Table 1 summarizes the results of the DD screen, 5' RACE, and comparisons of the deduced amino acid sequences to protein sequences in the National Center for Biotechnology Information (NCBI) databases.

The polypeptide encoded by full-length SD7C shares 88 and $45 \%$ amino acid identity with a mitochondrial transcription termination factor (mTERF)-related protein from Arabidopsis (NP-181009) and Solanum demissum (AAW28562), respectively (Figure 2, Table 1). The open reading frame of SD7C codes for a protein of 302 amino acid residues with a calculated molecular mass of $34 \mathrm{kDa}$ and a predicted isoelectric point of 8.9. A search of conserved domains within the SD7C sequence revealed the presence of a single mTERF domain between amino acid residues 40 and 288 (http://www.ncbi.nlm.nih.gov/Structure/cdd/wrpsb.cgi). 


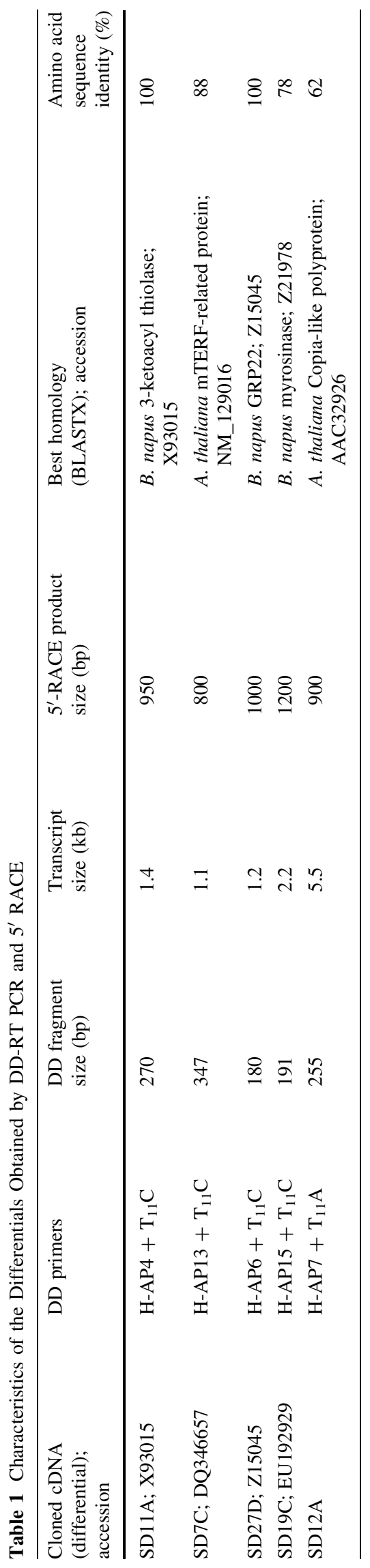

The SD27D (1071 bp) encodes a previously annotated glycine-rich protein22 (GRP22) of 291 amino acids that contains a secretory pathway signal peptide cleavage site between residues 21 and 22 . A search for closely related orthologs identified a single protein in both Arabidopsis (AAN15432) and rice (BAD30592) with amino acid identities of 83 and $57 \%$, respectively.

The protein encoded by the partial SD19C (1381 bp) was found to be most closely related to myrosinase Myr2.Bn1 of B. napus (Thangstad and others 1993), displaying $78 \%$ amino acid identity with that protein (Table 1). The sequence alignment of SD19C with three subfamilies of B. napus myrosinases, MA, MB and MC, is shown in Figure 3. Because the encoded polypeptide did not reveal $100 \%$ identity with any known myrosinase of $B$. napus, it is thought to represent a new member of the myrosinase gene family in $B$. napus.

The partial sequences of SD11A (1021 bp) and SD9B (280 bp) showed $100 \%$ identity at the nucleotide and amino acid level to a 3-ketoacyl-CoA thiolase of B. napus (Table 1). The SD9B cDNA contained an extra 32 nucleotides in its $3^{\prime}$ UTR, but was otherwise identical to SD11A; the $5^{\prime}$ sequence of SD9B therefore was not determined. The closest Arabidopsis ortholog (NP_180873, AT2G33150) shares $96 \%$ amino acid identity with the B. napus sequence.

The SD12A cDNA was isolated as a differential that showed higher expression in untreated seedlings compared with EBR-treated seedlings. The partial sequence of SD12A (1021 bp) shared $62 \%$ amino acid identity with a copia-like retroelement polyprotein of Arabidopsis (Table 1).

Detailed Expression Analysis of the Differentially Expressed cDNAs

To determine which cDNAs were induced by EBR and which were induced by both EBR and stress, we examined the expression patterns of the above sequences at various time points, including before, during, and after a heat stress treatment. Total RNA was isolated from untreated and EBR-treated seedlings that were either maintained at $20^{\circ} \mathrm{C}$ or heat-stressed for $1,2,3$, or $4 \mathrm{~h}$ at $45^{\circ} \mathrm{C}$, and from seedlings that were heat-stressed at $45^{\circ} \mathrm{C}$ for $4 \mathrm{~h}$ and then allowed to recover at $20^{\circ} \mathrm{C}$ for 6,19 , or $24 \mathrm{~h}$. The $5^{\prime}$-RACE products of the differentially expressed cDNAs were used as probes in the Northern blot analysis. The blot was also hybridized with a ribosomal DNA fragment to determine equal RNA loading in each lane. The experiment was done three times and the overall patterns of transcript accumulation were found to be reproducible.

With the exception of 3-ketoacyl-CoA thiolase, transcripts of all other genes detected at normal growing conditions were higher in EBR-treated seedlings compared 


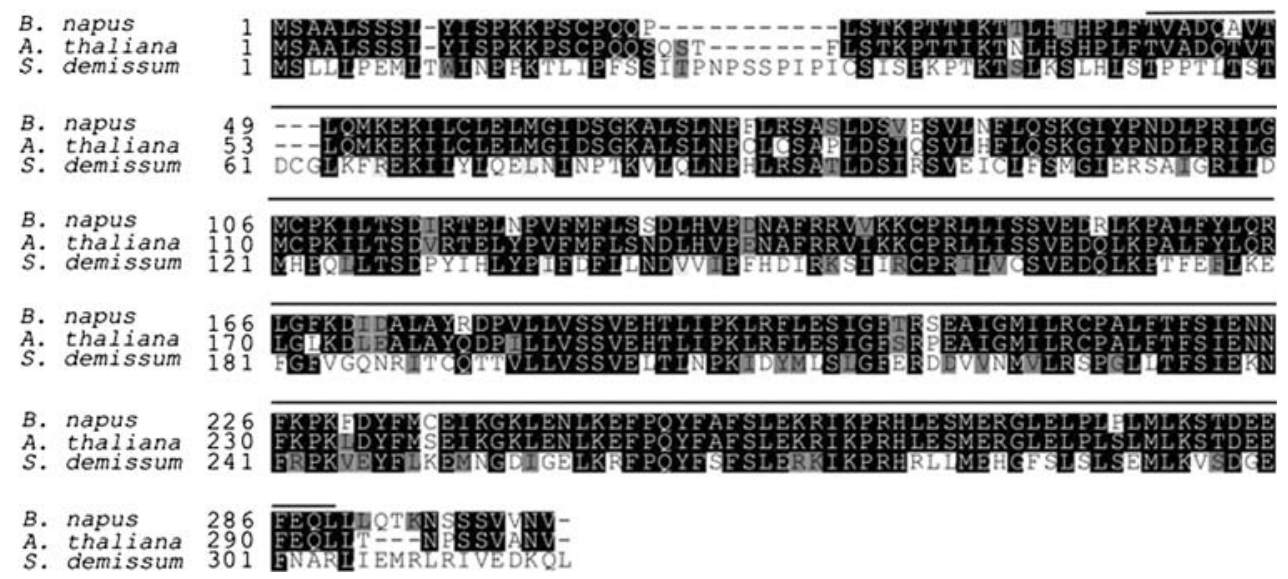

Fig. 2 Sequence analysis of SD7C cDNA encoding an mTERFrelated protein. Alignment of the deduced amino acid sequence of SD7C from B. napus (this study, accession number DQ346657) with related sequences from A. thaliana (accession number NP_181009) and Solanum demissum (accession number AAW28562) using ClustalW alignment. The ClustalW alignment results were imported to the BOXSHADE 3.21 program to obtain the shading of multiplealignment output. Identical amino acids are shown in the dark box and similar amino acids are indicated by the gray box. Dashes indicate gaps introduced to maximize alignment. The mTERF motif in $B$. napus sequence is shown by the black line on top of the sequence
Fig. 3 Comparison of the deduced partial amino acid sequence of SD19C with amino acid sequences of $B$. napus myrosinases belonging to three different subfamilies. Amino acid sequences of SD19C, MA (Myr1.Bn1, accession number Z21977), MB (Myr2.Bn1, accession number Z21978), and MC (accession number X79080) were compared using the ClustalW multiple sequence alignment program. Identical amino acids are shown in the dark box and similar amino acids are indicated by the gray box. Dashes indicate gaps introduced to maximize alignment

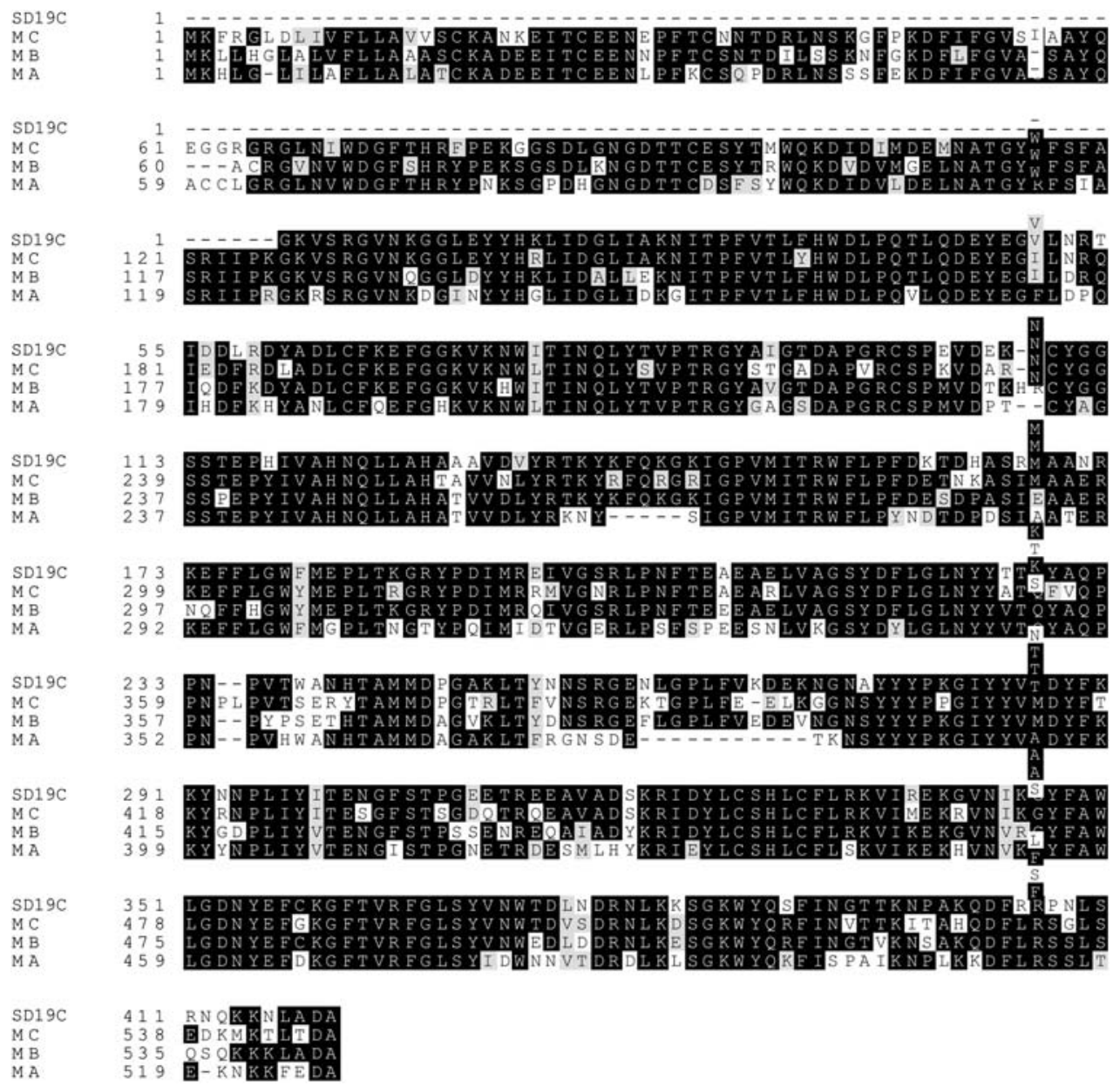

with untreated seedlings (Figure 4). The mTERF-related protein, GRP22, and myrosinase mRNAs were estimated at approximately two-, four-, and sixfold higher levels, respectively, in EBR-treated seedlings before heat stress. Transcripts encoding the mTERF-related protein accumulated to slightly higher levels at $1 \mathrm{~h}$ of heat stress, declining 


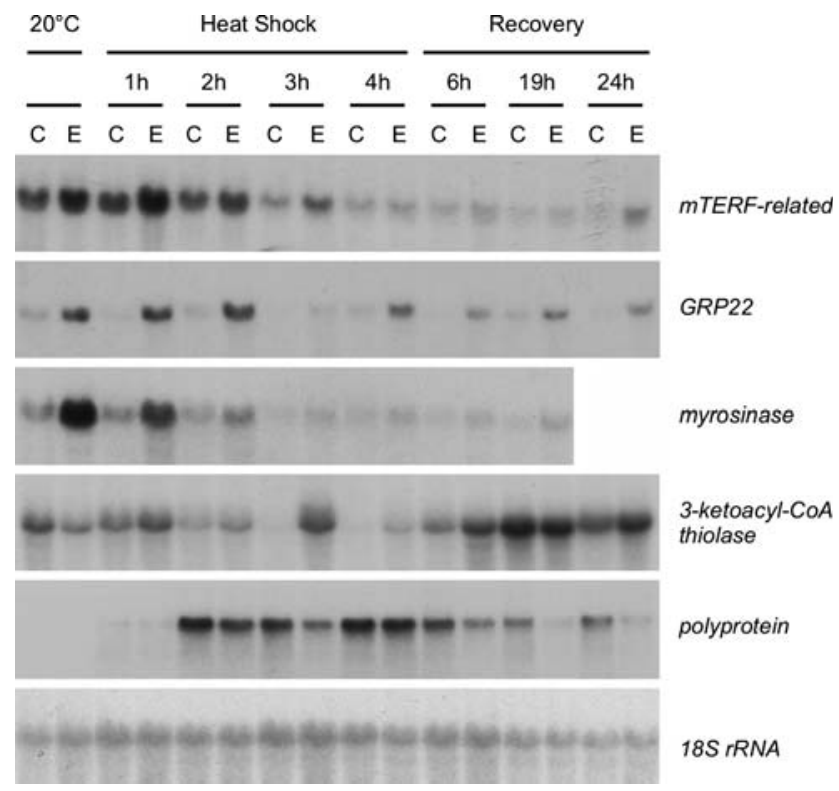

Fig. 4 Expression patterns of the differentials before, during, and after heat stress. Seedlings grown for 14 days in the absence (C) or presence (E) of $1 \mu \mathrm{M}$ EBR were exposed to $45^{\circ} \mathrm{C}$ for $1,2,3$, and $4 \mathrm{~h}$. Seedlings exposed to $45^{\circ} \mathrm{C}$ for $4 \mathrm{~h}$ were allowed to recover at $20^{\circ} \mathrm{C}$ for 6,19 , and $24 \mathrm{~h}$. Total RNA $(15 \mu \mathrm{g})$ was separated on denaturing formaldehyde agarose gels, blotted onto membranes, and hybridized with radiolabeled cDNA fragments (cloned $5^{\prime}$-RACE products). Hybridization of the blot with an 18S rRNA gene fragment was used to indicate equal RNA loading

progressively thereafter to $24 \mathrm{~h}$ recovery when the levels began to rise again in the treated seedlings. The GRP22 mRNA levels in treated seedlings also increased slightly in response to heat stress (compare lanes $1 \mathrm{~h}$ and $2 \mathrm{~h}$ ), declined by $3 \mathrm{~h}$, then rose again at $4 \mathrm{~h}$, but not to the original level. At all time points of the experiment (before, during, and after heat stress), GRP22 transcript expression was higher in EBR-treated seedlings versus untreated seedlings. The expression of myrosinase transcripts was repressed with increasing hours of heat stress and during the recovery period, but significantly higher levels were observed in EBR-treated seedlings before stress. Thus, in the cases of mTERF-related protein, GRP22, and myrosinase, the transcript levels remained higher in EBR-treated seedlings throughout, although a decline in the overall expression occurred during prolonged heat stress and recovery. The slight increase in the expression of mTERFrelated protein and GRP22 mRNAs at earlier time points of heat stress was more obvious in EBR-treated samples.

In contrast to these genes, 3-ketoacyl-CoA thiolase mRNA levels were higher in untreated seedlings before stress, but for most time points during stress and recovery periods the expression was elevated in treated seedlings. The levels peaked at $19 \mathrm{~h}$ of recovery in both sets of plants. A maximum difference of approximately 12 -fold in transcript levels between the two sets of plants was observed at $3 \mathrm{~h}$ of heat stress.

Unlike other differentials, transcripts encoding a copialike retroelement polyprotein were not detected before heat stress, but were observed only after $2 \mathrm{~h}$ of heat stress. In accordance with its isolation as a differential that is expressed at higher levels in untreated seedlings, the retroelement polyprotein transcript levels were higher in untreated seedlings than in EBR-treated seedlings during heat stress and recovery periods. The pattern of expression of the polyprotein (SD12A) transcripts by Northern blotting thus faithfully matched the initial DD banding pattern.

Time Course Expression of the cDNAs in Response to EBR Treatment

Because the differentials were isolated from $B$. napus seedlings that were grown on EBR-containing medium for 14 days, we studied the accumulation kinetics of a subset of differentially expressed cDNAs (myrosinase and GRP22) in response to EBR treatment. Total RNA was isolated from $B$. napus seedlings grown first in a liquid nutrient medium in Magenta vessels for 7 days, and then treated with either $0.01 \%$ absolute ethanol or $1 \mu \mathrm{M}$ EBR for a varying number of days. The uptake of EBR was only through roots. Initially, the transcript levels were checked at 2, 6, 12, 18, 24, 30, 36, and $48 \mathrm{~h}$ after treatment with EBR. No significant differences in the levels of myrosinase and GRP22 transcripts could be detected after up to $48 \mathrm{~h}$ of treatment with EBR (data not shown); however, following 3 days of exposure to EBR, an approximately sevenfold increase in myrosinase transcripts and a fivefold increase in GRP22

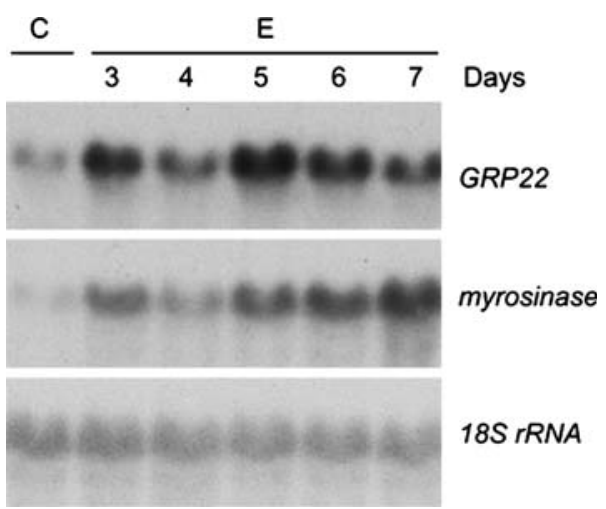

Fig. 5 Time course expression of GRP22 and myrosinase transcripts in response to EBR treatment. Total RNA $(15 \mu \mathrm{g})$ isolated from $B$. napus seedlings that were grown in liquid nutrient medium for 7 days in the absence of EBR and then exposed to either $0.01 \%$ absolute ethanol (C) or $1 \mu \mathrm{M}$ EBR (E) for different time periods was analyzed by Northern blot analysis. Control (C) represents seedlings treated with $0.01 \%$ ethanol for 7 days. Radiolabeled SD27D and SD19C ( $5^{\prime}$ RACE products) and 18S rRNA cDNA fragments were used as molecular probes 
was observed (Figure 5). Maximum accumulation of myrosinase and GRP22 transcripts occurred after 7 and 5 days, respectively. The control (lane $\mathrm{C}$ ) represents seedlings treated with $0.01 \%$ ethanol for 7 days. The expression of $B R U 1$, which is known to increase in response to $\mathrm{BR}$ in etiolated soybean epicotyls (Zurek and Clouse 1994), was also studied in B. napus seedlings. Transcript levels of the B. napus BRU1 ortholog increased only slightly within $24 \mathrm{~h}$ of EBR treatment, but a more pronounced increase was evident after 6 days of exposure to EBR (data not shown).

\section{In silico Gene Expression Analysis of the Arabidopsis} Orthologs

When this work was initiated, information on the transcriptome of Arabidopsis covering numerous data sets was not available. The availability of the AVT now allows data generation that serves as a key reference for research with other plants, especially for which microarrays with a limited set of gene-specific probes are available. We identified Arabidopsis orthologs closest to the B. napus differentials and examined their developmental and stress and hormoneresponsive expression using AVT. The transcripts of the mTERF-related protein from Arabidopsis (NP-181009) are expressed predominantly in young leaves and are maximally induced by $1 \mathrm{~h}$ of heat stress compared with other stresses (Figure 6A, right and left panels, respectively). The Arabidopsis GRP22 ortholog (AAN15432) is expressed mainly in the roots and hypocotyls of the plant and it is slightly induced by heat stress (Figure 6B, right and left panels, respectively). The Arabidopsis myrosinase ortholog TGG1 (NP_851077, 70\% amino acid identity) is not represented on the microarray chips used to generate expression profiling data, but the next closely related protein TGG2 (NP_568479, 66\% amino acid identity) is expressed at relatively higher levels in cotyledons, leaves, shoots, and flowers, and it is nearly absent from the roots (Figure 6C). No significant change in expression occurs in response to different abiotic stresses (not shown). The Arabidopsis 3-ketoacyl-CoA thiolase (NP_180873) mRNA is expressed in all tissues with highest levels being in seeds (Figure 6D). A minor induction takes place in response to drought (not shown).

None of the Arabidopsis orthologs showed induction by short-term treatment with BL or any other hormone. With the exception of the GRP22 ortholog, an insignificant increase was seen by treatment with ABA (data not shown).

\section{Discussion}

The identification of gene expression changes in response to BR treatment is imperative for defining the roles of BRs in various physiologic processes, including BR-mediated stress tolerance in plants. BR-mediated gene expression has been studied widely in Arabidopsis followed by studies in rice, tomato, and pea, but only a few such investigations have been reported for the agricultural genus Brassica, a close relative of Arabidopsis. We have previously shown that B. napus seedlings grown in the presence of $1 \mu \mathrm{M}$ EBR are significantly and reproducibly more heat tolerant than are untreated seedlings, and that this is in part due to higher accumulation of hsps in EBR-treated seedlings than in untreated seedlings (Dhaubhadel and others 1999). We have also demonstrated that increased accumulation of hsps in EBR-treated seedlings results from higher hsp synthesis, which correlates with increased expression of translational machinery components in these seedlings (Dhaubhadel and others 2002). Bearing in mind that EBR-induced gene expression changes are generally less than twofold (Vert and others 2005), we chose to use the differential display approach versus cross-hybridization of B. napus mRNA with Arabidopsis microarrays as a first step to investigate EBR-mediated gene expression changes in B. napus seedlings, beyond those associated with hsps. The key observations made with only a small subset of cDNAs characterized in this study are (1) the upregulation by EBR of genes involved in diverse physiologic processes such as fatty acid $\beta$-oxidation, glucosinolate metabolism, mitochondrial transcription, and possible cell wall strengthening; (2) a relatively high-level induction of gene expression in response to EBR (2-6-fold) in the absence of any stress; and (3) possible linkages of the newly identified genes with stress tolerance. At present, it is not known if the induction of these genes represents a primary or secondary response to EBR.

The DD screen was designed to identify cDNAs that are upregulated in EBR-treated seedlings both before and during stress. Detailed expression analysis of cDNAs encoding an mTERF-related protein, GRP22, and myrosinase revealed that transcripts corresponding to these cDNAs were expressed at higher levels in EBR-treated seedlings even prior to heat stress. The human mTERF is involved in the regulation of the transcription of the mitochondrial genome. Although a multizipper protein possessing three putative leucine zippers, this protein binds to DNA as a monomer (Fernandez-Silva and others 1997). Plants have numerous mTERF genes, with some genes predicted to be mitochondrial and others assigned to the chloroplast (Linder and others 2005). With the exception of a distant mTERF ortholog Mocl in Chlamydomonas reinhardtii, the loss of which causes a high light-sensitive phenotype (Schonfeld and others 2004), no functional information is available for the plant mTERFs. A leucine zipper domain could not be identified in the $B$. napus mTERF-related protein by a computational approach (Bornberg-Bauer and others 1998), but an intramitochondrial signal was identified in this 

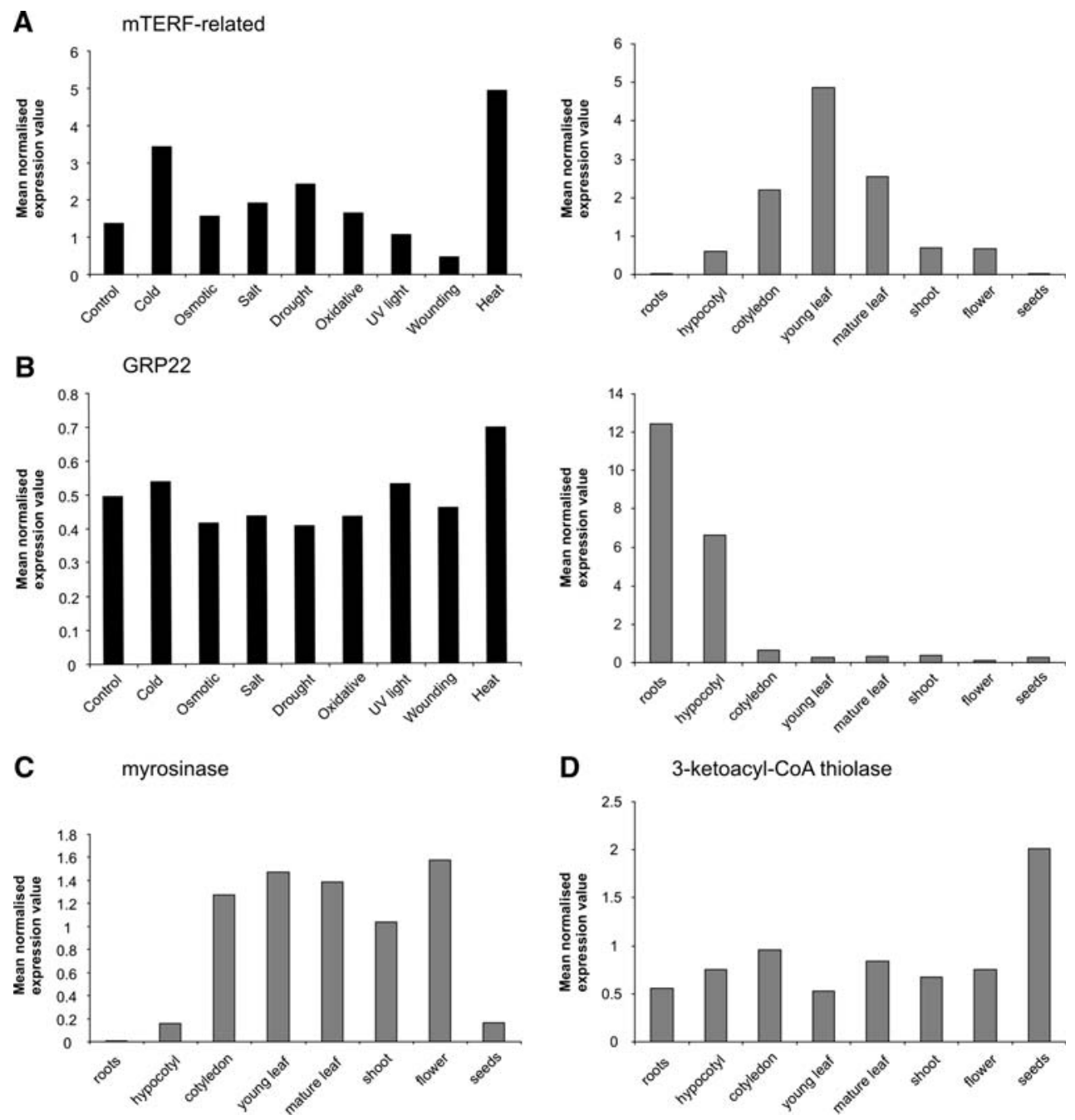

Fig. 6 In silico expression analysis of Arabidopsis orthologs of the $B$. napus differentials. The expression patterns of the Arabidopsis orthologs were generated using the AtGenExpress Visualization Tool (http://jsp.weigelworld.org/expviz/expviz.jsp). (A) Expression patterns of Arabidopsis mTERF-related protein (At2g34620) transcripts in response to abiotic stresses (left panel, black bars) and in different plant organs during development (right panel, gray bars). (B) Expression patterns of Arabidopsis GRP22 ortholog (At2g36120) transcripts in response to abiotic stresses (left panel, black bars) and in different plant organs during development (right panel, gray bars). (C) Transcript expression of Arabidopsis myrosinase ortholog

(At5g25980) during development. (D) Transcript expression of Arabidopsis 3-ketoacyl-CoA thiolase ortholog (At2g33150) during development. The stress conditions were heat, $1 \mathrm{~h}$ at $38^{\circ} \mathrm{C}$; cold, $1 \mathrm{~h}$ at $4^{\circ} \mathrm{C}$; osmotic, $300 \mathrm{mM}$ mannitol for $1 \mathrm{~h}$; salt, $150 \mathrm{mM} \mathrm{NaCl}$ for $1 \mathrm{~h}$; drought, 15 min dry air stream followed by incubation in climate chamber for $1 \mathrm{~h}$; oxidative, $10 \mathrm{M}$ methyl viologen for $1 \mathrm{~h}$; UV light, $0.25 \mathrm{~h} \mathrm{UV}-\mathrm{B}$ light field for $1 \mathrm{~h}$; wounding, leaf punctuation and tissues collected after $1 \mathrm{~h}$. The plant organs during the development were roots (7 day), hypocotyls (7 day), cotyledon ( 7 day), young leaves ( 7 day, first and second leaf), mature leaves (15 day), shoot (7 day), flower (28 day), seeds (stage 10)

protein. It remains to be seen if the higher expression of mTERF-related protein transcripts in EBR-treated B. napus seedlings compared with untreated seedlings can be linked to more efficient mitochondrial transcription and translation.

GRPs have been implicated in several independent physiologic processes based on their diverse expression patterns, subcellular localization, and structural characteristics (Sanchetto-Martins and others 2000). Expression of GRP mRNAs is influenced by heavy metals, low temperature, wounding, abscisic acid, and pathogen attack

(Sanchetto-Martins and others 2000). Some extremely glycine-rich GRPs (with approximately $70 \%$ glycine residues) act to strengthen the cell wall (Sakuta and others 1998). The level of glycine in GRP22 is relatively high (62\%), suggesting that it may have a role in cell wall strengthening. The predominant expression of the Arabidopsis ortholog in roots and hypocotyls of the plant (Figure 6B) supports this notion.

The slight increases in the transcript levels of B. napus mTERF-related protein and GRP22 in response to heat 
(Figure 4) parallel the in silico expression patterns generated for the Arabidopsis orthologs, which also show slight induction by heat stress (Figure 6A, B), but none of the Arabidopsis orthologs seem to be induced by short-term treatment with BR or other phytohormones (data not shown). The EBR effects on any one category of genes, however, can be quite different in Arabidopsis and $B$. napus. For instance, differences in the hsp transcript levels in EBR-treated and untreated seedlings are distinct in $B$. napus (Dhaubhadel and others 1999) but are subtle in Arabidopsis (Kagale and others 2007). Similarly, a subset of cold-regulated genes showed no difference in expression levels in EBR-treated and untreated seedlings of B. napus, but clear-cut differences were seen in Arabidospis seedlings (Kagale and others 2007). These results suggest that care should be exercised in extrapolating BR-induced gene expression patterns in Arabidopsis to other plant species.

Myrosinases (thioglucosidases) catalyze the hydrolysis of glucosinolates, a category of secondary products present primarily in the species of the order Capparales, releasing toxic products like nitriles, isothiocyanates, thiocyanates, epithionitriles, and oxazolidine-2-thiones. Glucosinolates and their breakdown products are believed to play a role in resistance to insects, and possibly other pathogens (Rask and others 2000). Myrosinases are encoded by a multigene family in B. napus that consists of three subfamilies: MA (Myr1), MB (Myr2), and MC. The polypeptide encoded by SD19C is most closely related to Myr2.Bn1 of B. napus (Thangstad and others 1993), which belongs to the MB subfamily. Members of the MB subfamily are expressed in the vegetative parts of the plant, whereas MA and MC subfamilies are expressed only in seeds (Lenman and others 1993; Falk and Rask 1995). SD19C was derived from mRNA isolated from the vegetative plant parts, and so we conclude that SD19C is a new member of the MB subfamily. A closely related Arabidopsis ortholog also shows higher expression in vegetative tissues of the plant compared to seeds (Figure 6C). Myrosinase is considered to be a defense-related enzyme against insect and pathogen attack (Rask and others 2000), and because EBR-treated plants are reported to be more resistant to pathogens (Khripach and others 2000), it is possible that the higher level of myrosinase expression in EBR-treated plants is a contributing factor to that resistance.

Two of the upregulated cDNAs, SD11A and SD9B, correspond to a previously identified 3-ketoacyl-CoA thiolase of $B$. napus. Nucleotide sequence comparison between the two cDNAs showed that SD9B contained 32 extra nucleotides in its $3^{\prime}$ UTR. This difference is likely due to different poly(A) sites used for addition of the poly(A) tail on the 3-ketoacyl-CoA thiolase transcripts, as multiple poly(A) sites can be present in a given plant mRNA species (Wu and others 1995). Only one conserved
poly(A) signal could be detected in SD11A and SD9B (not shown). However, unlike in animal systems, in plants less conserved sequences can function in the formation of the $3^{\prime}$ ends of mRNAs (Wu and others 1995; Rothnie 1996). Two semiconserved poly(A) signal sequences (TATAAT or AATGAT) were identified in SD9B, one of which may be responsible for the alternate poly(A) site.

The enzyme 3-ketoacyl-CoA thiolase catalyzes the terminal step in fatty acid $\beta$-oxidation during germination, releasing acetyl $\mathrm{CoA}$ that enters into the glyoxylate and citric acid cycles leading to gluconeogenesis. The biological significance of $\beta$-oxidation is to provide biosynthetic precursors from stored lipids. Thiolases also catalyze the first step of mevalonate biosynthesis pathway, which in turn leads to synthesis of steroids, abscisic acid (ABA), cytokinin (side chain), and gibberellic acid $\left(\mathrm{GA}_{3}\right)$ (Hopkins 1995). Because the Arabidopsis ortholog closest to the protein encoded by SD11A and SD9B has been functionally linked with $\beta$-oxidation of fatty acids (Carrie and others 2007), it is extrapolated that higher levels of this enzyme in EBR-treated plants during stress and recovery may drive more energy production, leading to better survival after lethal heat stress.

Unlike other differentials, SD12A transcripts were detected only in response to heat stress and at higher levels in untreated seedlings. The amino acid sequence encoded by SD12A showed similarity to a copia-like retrotransposon polyprotein. Retrotransposons are mobile elements that move to new locations in genomes through RNA intermediates (Bennetzen 2000). Little is known so far about plant retrotransposons. Suggestions that environmental stresses may induce transposition have been put forth to explain observations such as transposition of the maize $B s 1$ element in plants infected with barley stripe mosaic virus (Johns and others 1989). If the retrotransposon polyprotein transcript levels are correlated with the degree of transposition in a plant, which if in turn is correlated with the level of stress being experienced, then the comparatively lower levels of SD12A transcripts in EBR-treated seedlings would imply that these seedlings experience the damaging effects of heat stress to a lesser extent than do untreated seedlings.

The DD screen was performed with seedlings that were grown in the presence of EBR for 14 days. We tested the speed of response of GRP22 and myrosinase expression to EBR treatment. A significant increase in transcript levels first became evident after 3 days of treatment with EBR (Figure 5). It appears that the effect of EBR on the expression of these genes, at the levels seen in Figure 4, is not obtained with short-time exposure to EBR. Because BRs interact with other hormones (Yi and others 1999; Mussig and others 2000), one possibility is that a signaling pathway mediated by another hormone is involved in the 
downstream expression of GRP22 and myrosinase. Alternatively, it is possible that EBR directly regulates the expression of these genes and that the timing of increase in their expression is correlated with the time (between 48 and $72 \mathrm{~h}$ ) of uptake and transport of EBR in B. napus seedlings. Transport of BRs has been reported to occur from within hours to 7 days depending on the site of application and where it is monitored (Adam and Schneider 1999). In addition, the rate of transport varies with different plant species. Yet another possibility is that BRs act mainly during critical periods in development when the target cells or organs are sensitive to this class of growth regulators (Amzallag 2001). Except for a few genes, the mechanism of induction for most BR-regulated genes has not been described (Vert and others 2005). Thus, although direct or indirect control of EBR on the genes studied here and elsewhere remains to be defined, our interest is focused first on generating a comprehensive view of the molecular changes in seedlings treated with EBR.

In conclusion, the results of the present study clearly establish that EBR treatment leads to upregulation of genes linked with diverse responses. The literature suggests that higher expression of GRP22 and myrosinase may make plants more resistant to stresses. The significant increase in 3-ketoacyl-CoA thiolase transcript levels in treated seedlings during heat stress is likely to provide a metabolic advantage to these seedlings over untreated ones, and an increase in transcripts corresponding to mTERF-related protein in treated seedlings may aid in efficient transcription and translation in the mitochondria. Our previous results showing upregulation of the translational machinery (Dhaubhadel and others 2002) together with the results presented here unequivocally point to the need for further research into EBR-mediated molecular changes in $\mathrm{B}$. $\mathrm{na}$ pus, which no doubt will also add to our understanding of how EBR mediates stress tolerance in this agriculturally important oil seed crop.

Acknowledgments We thank Professor M. Perry and B. Winkfein for helpful suggestions. This research was supported by research grants to P.K. from the Natural Sciences and Engineering Research Council of Canada. S.D. was a recipient of the Ontario Graduate Scholarship.

Open Access This article is distributed under the terms of the Creative Commons Attribution Noncommercial License which permits any noncommercial use, distribution, and reproduction in any medium, provided the original author(s) and source are credited.

\section{References}

Adam G, Schneider B (1999) Uptake, transport and metabolism. In: Sakurai A, Yokota T, Clouse SD (eds) Brassinosteroids steroidal plant hormones. Springer Verlag, Tokyo, pp 113-136
Altschul SF, Madden TL, Schaffer AA, Zhang J, Zhang Z, Miller W, Lipman DJ (1997) Gapped BLAST and PSI -BLAST: a new generation of protein database search programs. Nucleic Acids Res 25:3389-3402

Amzallag GN (2001) Data analysis in plant physiology: are we missing the reality? Plant Cell Environ 24:881-890

Belkhadir Y, Wang X, Chory J (2006) Brassinosteroid signaling pathway. Sci STKE 364:cm4

Bennetzen JL (2000) Transposable element contributions to plant gene and genome evolution. Plant Mol Biol 42:251-269

Bishop GJ (2003) Brassinosteroid mutants of crops. J Plant Growth Regul 22:325-335

Bornberg-Baur E, Rivals E, Vingron M (1998) Computational approaches to identify leucine zippers. Nucleic Acids Res 26:2740-2746

Carrie C, Murcha MW, Millar AH, Smith SM, Whelan J (2007) Nine 3-ketoayl-CoA thiolases (KATs) and acetoacetyl-CoA thiolases (ACATs) encoded by five genes in Arabidopsis thaliana are targeted either to peroxosomes or cytosol but not to mitochondria. Plant Mol Biol 63:97-108

Clouse SD, Sasse J (1998) Brassinosteroids: essential regulators of plant growth and development. Annu Rev Plant Physiol Plant Mol Biol 49:427-451

Dhaubhadel S, Chaudhary S, Dobinson KF, Krishna P (1999) Treatment with 24-epibrassinolide, a brassinosteroid, increases the basic thermotolerance of Brassica napus and tomato seedlings. Plant Mol Biol 40:333-342

Dhaubhadel S, Browning KS, Gallie DR, Krishna P (2002) Brassinosteroid functions to protect the translational machinery and heat shock protein synthesis following thermal stress. Plant $\mathbf{J}$ 29:681-691

Falk A, Rask L (1995) Characterization of a new myrosinase in Brassica napus. Plant Mol Biol 27:863-874

Fernandez-Silva P, Martinez-Azorin F, Micol V, Attardi G (1997) The human mitochondrial transcription termination factor (mTERF) is a multizipper protein but binds to DNA as a monomer, with evidence pointing to intramolecular leucine zipper interactions. EMBO J 16:1066-1079

Glisin V, Crkvenjakov R, Byus C (1974) Ribonucleic acid isolated by cesium chloride centrifugation. Biochemistry 13:2633-2637

Goda H, Shimada Y, Asami T, Fujioka S, Yoshida S (2002) Microarray analysis of brassinosteroid-regulated genes in Arabidopsis. Plant Physiol 130:1319-1334

Goetz M, Godt DE, Roitsch T (2000) Tissue-specific induction of the mRNA for an extracellular invertase isoenzyme of tomato by brassinosteroids suggests a role for steroid hormones in assimilate partitioning. Plant J 22:515-522

He K, Gou X, Yuan T, Lin H, Asami T, Yoshida S, Russell SD, Li J (2007) BAK1 and BKK1 regulate brassinosteroid-dependent growth and brassinosteroid-independent cell-death pathways. Curr Biol 17:1109-1115

Hopkins WG (1995) Introduction to Plant Physiology. John Wiley \& Sons, New York, pp 125-144

$\mathrm{Hu}$ Y, Bao F, Li J (2000) Promotive effect of brassinosteroids on cell division involves a distinct CycD3-induction pathway in Arabidopsis. Plant J 24:693-701

Jiang J, Clouse SD (2001) Expression of a plant gene with sequence similarity to animal TGF- $\beta$ receptor interacting protein is regulated by brassinosteroids and required for normal development. Plant J 26:35-45

Johns MA, Babcock MS, Fuerstenberg SM, Freeling M, Simpson RB (1989) An unusually compact retrotransposon in maize. Plant Mol Biol 12:633-642

Kagale S, Divi UK, Krochko JE, Keller WA, Krishna P (2007) Brassinosteroid confers tolerance in Arabidopsis thaliana and Brassica napus to a range of abiotic stresses. Planta 225:353-364 
Karlova R, de Vries SC (2006) Advances in understanding brassinosteroid signaling. Sci STKE 354:36

Khripach V, Zhabinskii V, de Groot A (2000) Twenty years of brassinosteroids: steroidal plant hormones warrant better crops for the XXI century. Ann Bot 86:441-447

Kilian J, Whitehead D, Horak J, Wanke D, Weinl S, Batistic O, D'Angelo C, Bornberg-Bauer E, Kudla J, Harter K (2007) The AtGenExpress global stress expression data set: protocols, evaluation and model data analysis of UV-B light, drought and cold stress responses. Plant J 50:347-363

Krishna P (2003) Brassinosteroid-mediated stress responses. J Plant Growth Regul 22:289-297

Lenman M, Falk A, Xue J, Rask L (1993) Characterization of a Brassica napus myrosinase pseudogene: myrosinases are members of the BGA family of $\beta$-glycosidases. Plant Mol Biol 21:463-474

Li J, Jin H (2007) Regulation of brassinosteroid signaling. Trends Plant Sci 12:37-41

Liang P, Averboukh L, Pardee AB (1993) Distribution and cloning of eukaryotic mRNAs by means of differential display: refinements and optimization. Nucleic Acids Res 21:3269-3275

Liang P, Pardee AB (1992) Differential display of eukaryotic messenger RNA by means of the polymerase chain reaction. Science 257:967-971

Linder T, Park CB, Asin-Cayuela J, Pellegrini M, Larsson NG, Falkenberg M, Samuelsson T, Gustafsson CM (2005) A family of putative transcription termination factors shared amongst metazoans and plants. Curr Genet 484:265-269

Mussig C, Biesgen C, Lisso J, Uwer U, Weiler EW, Altmann T (2000) A novel stress-inducible 12-oxophytodienoate reductase from Arabidopsis thaliana provides a potential link between brassinosteroid action and jasmonic acid synthesis. J Plant Physiol 157:143-152

Mussig C, Fischer S, Altmann T (2002) Brassinosteroid-regulated gene expression. Plant Physiol 129:1241-1251

Mussig C, Lisso J, Coll-Garcia D, Altmann T (2006) Molecular analysis of brassinosteroid action. Plant Biol (Stuttg) 8:291-296

Rask L, Andreasson E, Ekbom B, Eriksson S, Pontoppidan B, Meijer J (2000) Myrosinase: gene family evolution and herbivore defence in Brassicaceae. Plant Mol Biol 42:93-113
Rothnie HM (1996) Plant mRNA 3' end formation. Plant Mol Biol 32:43-61

Sakuta C, Oda A, Yamakawa S, Satoh S (1998) Root-specific expression of genes for novel glycine-rich proteins by use of an antiserum against xylem sap proteins of cucumber. Plant Cell Physiol 39:1330-1336

Sanchetto-Martins G, Franco LO, de Oliveira DE (2000) Plant glycine-rich proteins: a family or just proteins with a common motif? Biochim Biophys Acta 1492:1-14

Sasse JM (2003) Physiological actions of brassinosteroids: an update. J Plant Growth Regul 22:276-288

Schmid M, Davison TS, Henz SR, Pape UJ, Demar M, Vingron M, Schölkopf B, Weigel D, Lohmann JU (2005) A gene expression map of Arabidopsis thaliana development. Nat Genet 37:501506

Schönfeld C, Wobbe L, Borgstädt R, Kienast A, Nixon PJ, Kruse O (2004) The nucleus-encoded protein MOC1 is essential for mitochondrial light acclimation in Chlamydomonas reinhardtii. $\mathrm{J}$ Biol Chem 279:50366-50374

Thangstad OP, Winge P, Husebye H, Bones A (1993) The myrosinase (thioglucoside glucohydrolase) gene family in Brassicaceae. Plant Mol Biol 23:511-524

Vert G, Nemhauser JL, Geldner N, Hong F, Chory J (2005) Molecular mechanisms of steroid hormone signaling in plants. Annu Rev Cell Dev Biol 21:177-201

Wu L, Ueda T, Messing J (1995) The formation of mRNA $3^{\prime}$ ends in plants. Plant J 8:323-329

Yi HC, Joo S, Nam KH, Lee JS, Kang BG, Kim WT (1999) Auxin and brassinosteroid differentially regulate the expression of three members of the 1-aminocyclopropane-1-carboxylate synthase gene family in mung bean (Vigna radiata L.). Plant Mol Biol 41:443-454

Yin Y, Wang ZY, Mora-Garcia S, Li J, Yoshida S, Asami T, Chory J (2002) BES1 accumulates in the nucleus in response to brassinosteroids to regulate gene expression and promote stem elongation. Cell 109:181-191

Zurek DM, Clouse SD (1994) Molecular cloning and characterization of a brassinosteroid-regulated gene from elongating soybean epicotyls. Plant Physiol 104:161-170 